\title{
An Efficient Parallel Algorithm for Scheduling Interval Ordered Tasks
}

\author{
Yoojin Chung ${ }^{1}$, Kunsoo Park $^{2 \star}$, and Hyuk-Chul Kwon ${ }^{3 \star}$ \\ 1 Research Institute of Computer and Information \& Communication, \\ Pusan National University, Pusan, 609-735, Korea \\ chungyj@pusan.ac.kr \\ 2 School of Computer Science and Engineering, \\ Seoul National University, Seoul 151-742, Korea \\ kpark@theory.snu.ac.kr \\ 3 Division of Computer Science and Engineering, \\ Pusan National University, Pusan, 609-735, Korea \\ hckwon@pusan.ac.kr
}

\begin{abstract}
We present an efficient parallel algorithm for scheduling $n$ unit length tasks on $m$ identical processors when the precedence graphs are interval orders. Our algorithm requires $O\left(\log ^{2} v+(n \log n) / v\right)$ time and $O\left(n v^{2}+n^{2}\right)$ operations on the CREW PRAM, where $v \leq n$ is a parameter. By choosing $v=\sqrt{n}$, we obtain an $O(\sqrt{n} \log n)$-time algorithm with $O\left(n^{2}\right)$ operations. For $v=n / \log n$, we have an $O\left(\log ^{2} n\right)$ time algorithm with $O\left(n^{3} / \log ^{2} n\right)$ operations. The previous solution takes $O\left(\log ^{2} n\right)$ time with $O\left(n^{3} \log ^{2} n\right)$ operations on the CREW PRAM. Our improvement is mainly due to a reduction of the $m$-processor scheduling problem for interval orders to that of finding a maximum matching in a convex bipartite graph.
\end{abstract}

\section{Introduction}

The $m$-processor scheduling problem for a precedence graph $\boldsymbol{G}$ is defined as follows. An input graph $\boldsymbol{G}$ has $n$ vertices each of which represents a task to be executed on any one of $m$ identical processors. Each task requires exactly one unit of execution time on any processor. At any timestep at most one task can be executed by a processor. If there is a directed edge from task $t$ to task $t^{\prime}$, then task $t$ must be completed before task $t^{\prime}$ is started. An $m$-processor schedule for $\boldsymbol{G}$ specifies the timestep and the processor on which each task is to be executed. The length of a schedule is the number of timesteps in it. A solution to the problem is an optimal (i.e., shortest length) schedule for $\boldsymbol{G}$.

The $m$-processor scheduling problem for arbitrary precedence graphs has been studied extensively. When $m=2$, there are polynomial-time algorithms for the problem [6397, and when $m$ is part of the input, the problem is known to be NP-hard [20]. When $m$ is part of the input, several researchers have considered restrictions on the precedence graphs. Polynomial-time algorithms for the $m$ processor scheduling problem are known for the cases that the precedence graphs

* This work was supported by the Brain Korea 21 Project. 
are trees 12 and interval orders [15]. A survey of results on other special cases of the problem can be found in 13 .

In parallel computation, the two processor case has been studied mostly. When $m=2$, Helmbold and Mayr [11] gave the first NC algorithm and Vazirani and Vazirani [21] presented an RNC algorithm. Jung, Serna and Spirakis [16] developed an $O\left(\log ^{2} n\right)$-time algorithm using $O\left(n^{3} \log ^{2} n\right)$ operations on the CREW PRAM. When $m=2$ and the precedence graphs are interval orders, Moitra and Johnson [18 and Chung, Park and Cho 2] gave NC algorithms, and the one in 22 requires $O\left(\log ^{2} v+(n \log n) / v\right)$ time and $O\left(n v^{2}+n^{2}\right)$ operations on the CREW PRAM, where $v \leq n$ is a parameter.

When $m$ is part of the input and the precedence graphs are interval orders, Sunder and He 19 developed the first NC algorithm for the scheduling problem, which takes $O\left(\log ^{2} n\right)$ time using $O\left(n^{5} \log ^{2} n\right)$ operations or $O\left(\log ^{3} n\right)$ time using $O\left(n^{4} \log ^{3} n\right)$ operations on the priority CRCW PRAM. Mayr [14] gave an $O\left(\log ^{2} n\right)$-time algorithm using $O\left(n^{3} \log ^{2} n\right)$ operations on the CREW PRAM.

In this paper, we present an efficient parallel algorithm for the $m$-processor scheduling problem when the precedence graphs are interval orders. Our algorithm takes $O\left(\log ^{2} v+(n \log n) / v\right)$ time using $O\left(n v^{2}+n^{2}\right)$ operations on the CREW PRAM, where $v \leq n$ is a parameter. By choosing $v=\sqrt{n}$, we obtain an $O(\sqrt{n} \log n)$-time algorithm with $O\left(n^{2}\right)$ operations. For $v=n / \log n$, we have an $O\left(\log ^{2} n\right)$-time algorithm with $O\left(n^{3} / \log ^{2} n\right)$ operations.

We briefly compare Mayr's algorithm and ours. A parallel algorithm that computes the length of an optimal $m$-processor schedule for an interval order will be called an m-LOS algorithm. Mayr's algorithm basically consists of two parts. The first part uses an $m$-LOS algorithm to compute the lengths of optimal schedules, which takes $O\left(\log ^{2} n\right)$ time using $O\left(n^{3} \log ^{2} n\right)$ operations on the CREW PRAM. The second part computes an actual scheduling, which takes $O\left(\log ^{2} n\right)$ time using $O\left(n^{3} \log ^{2} n\right)$ operations on the CREW PRAM. Our algorithm also consists of two parts and its first part is an $m$-LOS algorithm, but our algorithm is quite different from Mayr's as follows.

- We give an efficient $m$-LOS algorithm that takes $O\left(\log ^{2} v+(n \log n) / v\right)$ time and $O\left(n v^{2}+n^{2}\right)$ operations on the CREW PRAM by generalizing the techniques used for two-processor scheduling in [2].

- After computing the lengths of optimal schedules, we reduce the $m$-processor scheduling problem for interval orders to that of finding a maximum matching in a convex bipartite graph using the lengths to compute an actual scheduling. Therefore, the part of computing an actual scheduling in our algorithm takes $O\left(\log ^{2} n\right)$ time using $O\left(n \log ^{2} n\right)$ operations on the EREW PRAM.

The remainder of this paper is organized as follows. The next section gives basic definitions and a sequential scheduling algorithm. Section 3 describes the reduction of $m$-processor scheduling to maximum matching in a convex bipartite graph. Section 4 describes our efficient $m$-LOS algorithm. 


\section{Basic Definitions and Sequential Algorithm}

In this section we describe basic definitions and a sequential $m$-processor scheduling algorithm. An instance of the $m$-processor scheduling problem is given by a precedence graph $\boldsymbol{G}=(V, E)$. A precedence graph is an acyclic and transitively closed digraph. Each vertex of $\boldsymbol{G}$ represents a task whose execution requires unit time on one of $m$ identical processors. If there is a directed edge from task $t$ to task $t^{\prime}$, then task $t$ must be completed before task $t^{\prime}$ is started. In such a case, we call $t$ a predecessor of $t^{\prime}$ and $t^{\prime}$ a successor of $t$. We use $\left\langle t, t^{\prime}\right\rangle$ to denote a directed edge from $t$ to $t^{\prime}$. A schedule is a mapping from tasks to timesteps such that at most $m$ tasks are mapped to each timestep and for every edge $\left\langle t, t^{\prime}\right\rangle, t$ is mapped to an earlier timestep than $t^{\prime}$. The length of a schedule is the number of timesteps used. An optimal schedule is one with the shortest length.

Let $I=\left\{I_{1}, \ldots, I_{n}\right\}$ be a set of intervals with each interval $I_{i}$ represented by $I_{i} . l$ and $I_{i} . r$, where $I_{i} . l$ and $I_{i} . r$ denote the left and right endpoints of interval $I_{i}$, respectively. Without loss of generality, we assume that all the endpoints are distinct. We also assume that the intervals are labeled in the increasing order of right endpoints, i.e., $I_{1} . r<I_{2} . r<\cdots<I_{n}$.r because sorting can be done in $O(\log n)$ time using $O(n \log n)$ operations on the EREW PRAM [4]. Given a set $I$ of $n$ intervals, let $\boldsymbol{G}_{\boldsymbol{I}}=(V, E)$ be a graph such that

- $V=I=\left\{I_{1}, I_{2}, \ldots, I_{n}\right\}$ and

$-E=\left\{\left\langle I_{i}, I_{j}\right\rangle \mid 1 \leq i, j \leq n\right.$ and $\left.I_{i} . r<I_{j} . l\right\}$.

Such a graph $\boldsymbol{G}_{\boldsymbol{I}}$ is called an interval order. Note that $\boldsymbol{G}_{\boldsymbol{I}}$ is a precedence graph. Given a set $I$ of $n$ intervals, the interval graph $G_{I}$ is an undirected graph such that each vertex corresponds to an interval in $I$ and two vertices are adjacent whenever the corresponding intervals have at least one point in common. Therefore, an interval graph $G_{I}$ is a complement of the interval order $\boldsymbol{G}_{\boldsymbol{I}}$. We say that two vertices are independent if they are not adjacent in a graph. Note that overlapping intervals are adjacent in $G_{I}$ and they are independent of each other in $\boldsymbol{G}_{\boldsymbol{I}}$. In what follows, we use the words tasks and intervals interchangeably.

A schedule of length $r$ on $m$ processors for an interval order $\boldsymbol{G}_{\boldsymbol{I}}$ can be represented by an $m \times r$ matrix $M$, where the columns are indexed by $1, \ldots, r$ and the rows are indexed by $1, \ldots, m$. Let $P_{1}, \ldots, P_{m}$ denote the $m$ identical processors. If task $x$ is scheduled on processor $P_{i}$ at timestep $\tau$, then $x$ is assigned to a slot $M[i, \tau]$. No two tasks are assigned to the same slot in $M$. A slot of $M$ to which no task is assigned is said to have an empty task. We assume that the right endpoint of an empty task is larger than all right endpoints in $I$. A column of $M$ is called full if it does not have an empty task. Let opt(I) be the length of an optimal schedule for an interval order $\boldsymbol{G}_{\boldsymbol{I}}$.

\section{Algorithm m-seq $(I, m)$}

Input: intervals in $I$

Output: $m \times \operatorname{opt}(I)$ matrix $M_{s}$ 
begin

$\tau \leftarrow 1$;

$S_{\tau} \leftarrow$ the list of intervals in $I$ sorted in the increasing order of right endpoints;

while $S_{\tau} \neq \phi$ do

$S^{\prime} \leftarrow\{\}$

Extract the first interval from $S_{\tau}$ and insert it to $S^{\prime}$;

repeat

Scan $S_{\tau}$ from left to right. When interval $w$ is scanned,

if $w$ is overlapping every interval in $S^{\prime}$

then extract $w$ from $S_{\tau}$ and insert it to $S^{\prime}$ fi;

until ( $S^{\prime}$ contains $m$ intervals or all intervals of $S_{\tau}$ are considered)

Schedule the intervals of $S^{\prime}$ in column $\tau$ of $M_{s}$

in the order of the elements in list $S^{\prime}$;

$S_{\tau+1} \leftarrow S_{\tau}$

$\tau \leftarrow \tau+1$

od

Output the schedule $M_{s}$ constructed;

end

Fig. 1. Sequential scheduling algorithm

The sequential algorithm 15 in Figure 1 solves the $m$-processor scheduling problem for an interval order $\boldsymbol{G}_{\boldsymbol{I}}$, which runs in $O(n \log n)$ time. Let $I(1, j)$ denote $\left\{I_{1}, \ldots, I_{j}\right\}, 1 \leq j \leq n$. Note that m-seq computes an optimal schedule for $\boldsymbol{G}_{\boldsymbol{I}(\mathbf{1}, \boldsymbol{j})}$. We can easily get the following facts from algorithm m-seq.

Fact 1 All the intervals in the same column of $M_{s}$ overlap each others.

Fact 2 In each column $\tau$ of $M_{s}$ in m-seq, $M_{s}[1, \tau] \cdot r \leq M_{s}[2, \tau] \cdot r \leq \ldots \leq$ $M_{s}[m, \tau] . r$.

Fact 3 In the first row of $M_{s}, M_{s}[1,1] . r<M_{s}[1,2] . r<\ldots<M_{s}[1, m] . r$.

Proof. It follows from the fact that for every $\tau, M_{s}[1, \tau]$ is the first ending interval in $S_{\tau}$ and $M_{s}\left[1, \tau^{\prime}\right]$ with $\tau^{\prime}>\tau$ is in $S_{\tau}$.

\section{Constructing an Optimal Schedule}

In this section we describe our parallel $m$-processor scheduling algorithm for interval orders. We first describe characteristics of maximal cliques in interval graphs. A set of intervals form a clique if each pair of intervals in the set has a nonempty intersection. If we scan any given interval $x$ from its left endpoint to its right, we can meet all those maximal cliques to which $x$ belongs. This yields the Gilmore-Hoffman theorem [10]. 

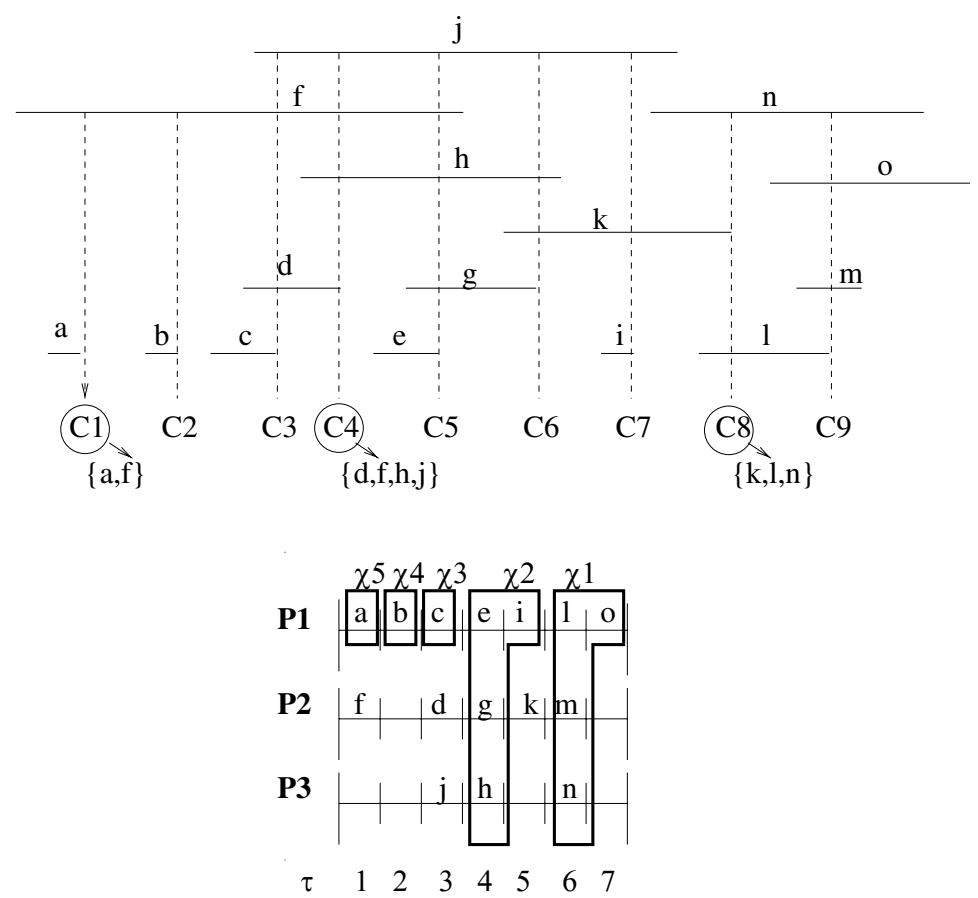

Fig. 2. An interval set $I$ and $\boldsymbol{G}_{\boldsymbol{I}}$ 's optimal schedule when $m=3$.

Theorem 1. 10. The maximal cliques of an interval graph can be linearly ordered so that for any given interval $x$, the set of cliques in which $x$ occurs appear consecutively in the linear order.

Let $k$ be the number of maximal cliques in $G_{I}$. Let $C_{1}, \ldots, C_{k}$ be the maximal cliques of $G_{I}$ in the ordering of Theorem 1 Given an interval set, we can find the maximal cliques of the interval graph $G_{I}$ using Lemma 1 In Figure 2, dotted vertical lines mark the right endpoints of Lemma 1 i.e., there are nine maximal cliques in $G_{I}$ and they are $C_{1}=\{a, f\}, C_{2}=\{b, f\}, C_{3}=\{c, d, f, j\}$, etc.

Lemma 1. 2. In an interval set $I$, a right endpoint represents a maximal clique of $G_{I}$ if and only if its previous endpoint in the sorted list of left and right endpoints is a left endpoint.

For each interval $x \in I$, let $s_{x}$ and $l_{x}$ be the smallest and the largest $j$, respectively, such that $x$ belongs to $C_{j}$. In Figure $2 s_{h}=4$ and $l_{h}=6$ because interval $h$ is in $C_{4}, C_{5}$ and $C_{6}$. Let $\operatorname{sltask}(i, j), 1 \leq i, j \leq k$, be the set of intervals $x$ such that $i \leq s_{x}$ and $l_{x} \leq j$. In Figure 2, $\operatorname{sltask}(1,5)=\{a, b, c, d, e, f\}$. Note that algorithm m-seq $(I, m)$ in Figure 1 computes an optimal schedule for $\boldsymbol{G}_{\boldsymbol{s l t a s k}(\mathbf{1}, j)}$, $1 \leq j \leq k$, because m-seq computes an optimal schedule for $\boldsymbol{G}_{\boldsymbol{I}(\mathbf{1}, t)}, 1 \leq t \leq n$, and maximal cliques $C_{1}, \ldots, C_{k}$ of $G_{I}$ are labeled by scanning endpoints of $I$ 
from left to right using Lemma 1 Let $\operatorname{len}(i, j)$ be the minimum number of timesteps required to schedule all tasks in $\operatorname{sltask}(i, j)$, i.e., $\operatorname{opt}(\operatorname{sltask}(i, j))$.

Lemma 2. 2] For two intervals $x, y \in I, l_{x}<s_{y}$ if and only if $x . r<y . l$.

We now describe our parallel $m$-processor scheduling algorithm for interval orders. Our algorithm consists of two parts. The first part is an $m$-LOS algorithm m-length, which will be described in Section 4. Algorithm m-length computes len $(1, j)$ for all $1 \leq j \leq k$. The second part computes an optimal schedule by reducing the $m$-processor scheduling problem for an interval order to that of finding a maximum matching in a convex bipartite graph.

We first describe the definition of a convex bipartite graph. A convex bipartite graph $G$ is a triple $(A, B, E)$ such that $A=\left\{a_{1}, a_{2}, \ldots, a_{n}\right\}$ and $B=$ $\left\{b_{1}, b_{2}, \ldots, b_{m}\right\}$ are disjoint sets of vertices and the edge set $E$ satisfies the following properties:

(1) Every edge of $E$ is of the form $\left(a_{i}, b_{j}\right)$.

(2) If $\left(a_{i}, b_{j}\right) \in E$ and $\left(a_{i}, b_{j+t}\right) \in E$, then $\left(a_{i}, b_{j+r}\right) \in E$ for every $1 \leq r<t$.

Property (1) is a bipartite property while property (2) is a convexity property. It is clear that every convex bipartite graph $G=(A, B, E)$, where $A=$ $\left\{a_{1}, \ldots, a_{n}\right\}$ and $B=\left\{b_{1}, \ldots, b_{m}\right\}$, is uniquely represented by a set of triples: $T=\left\{\left(a_{i}, g_{i}, h_{i}\right) \mid 1 \leq i \leq n\right\}$, where $g_{i}=\min \left\{j \mid\left(a_{i}, b_{j}\right) \in E\right\}$ and $h_{i}=\max \left\{j \mid\left(a_{i}, b_{j}\right) \in E\right\}$. Dekel and Sahni [5] developed an $O\left(\log ^{2} n\right)$-time convex bipartite maximum matching algorithm using $O\left(n \log ^{2} n\right)$ operations on the EREW PRAM.

Our $m$-processor scheduling algorithm is as follows.

\section{Algorithm m-schedule}

- Step 1: Compute $s_{x}$ and $l_{x}$ for every $x \in I$.

- Step 2: Let $L_{0}=0$. Let $L_{j}=\operatorname{len}(1, j)$ for $1 \leq j \leq k$ and compute $L_{j}$.

- Step 3: Construct a convex bipartite graph $G_{b}=\left(A_{b}, B_{b}, E_{b}\right)$, where $A_{b}=I$, $B_{b}=\left\{1,2, \ldots, m L_{k}\right\}$ and $E_{b}$ is computed from $L_{j}, j \leq k$, as follows. If an interval $x \in I$ is in a maximal clique $C_{t}$ in $G_{I}$, then $x$ is adjacent to all $j$ in $B_{b}$ such that $m L_{t-1}+1 \leq j \leq m L_{t}$. Since an interval $x$ is in every $C_{t}$ such that $s_{x} \leq t \leq l_{x}$ by Theorem 1, $G_{b}$ is represented by $T=\left\{\left(x, m L_{s_{x}-1}+1, m L_{l_{x}}\right) \mid\right.$ $x \in I\}$.

- Step 4: Find a maximum matching in $G_{b}$. Then an optimal schedule for $\boldsymbol{G}_{\boldsymbol{I}}$ is represented by an $m \times L_{k}$ matrix $M_{b}$, whose $j$-th column consists of the tasks in $A_{b}$ matched with $m(j-1)+1, \ldots, m j$ in $B_{b}$ in the maximum matching of $G_{b}$.

We now prove the correctness of algorithm m-schedule.

Lemma 3. All the intervals in the same column of $M_{b}$ are independent of each other in $\boldsymbol{G}_{\boldsymbol{I}}$. 
Proof. By definition of $G_{b}$, all intervals that are adjacent to one of $m L_{j-1}+$ $1, \ldots, m L_{j}$ in $G_{b}, 1 \leq j \leq L_{k}$, are also adjacent to all of $m L_{j-1}+1, \ldots, m L_{j}$ and they are all in the same maximal clique in $G_{I}$. Therefore, the intervals matched with $m L_{j-1}+1, \ldots, m L_{j}$ in the maximum matching of $G_{b}$ are independent of each other in $\boldsymbol{G}_{\boldsymbol{I}}$. Since all the intervals in columns $L_{j-1}+1, \ldots, L_{j}, 1 \leq j \leq k$, in $M_{b}$ are independent of each other in $\boldsymbol{G}_{\boldsymbol{I}}$, we have the lemma.

Lemma 4. The convex bipartite graph $G_{b}=\left(A_{b}, B_{b}, E_{b}\right)$ has a maximum matching of size $n$, i.e., all intervals in $A_{b}$ are matched in a maximum matching of $G_{b}$.

Proof. Construct an edge set $E^{\prime} \subseteq A_{b} \times B_{b}$ from $M_{s}$ constructed by algorithm m-seq in Figure 1 as follows. $E^{\prime}=\left\{(x, j) \mid x \in A_{b}\right.$ is the $j$-th element of $M_{s}$ in the column-major order $\}$. Then every edge $(x, j)$ in $E^{\prime}$ satisfies $m(\tau-1)+1 \leq$ $j \leq m \tau$, where $\tau$ is the column number in $M_{s}$ at which $x$ is. We first show that $E^{\prime} \subseteq E_{b}$. Note that $\tau \leq L_{l_{x}}$ because m-seq produces an optimal schedule for $\boldsymbol{G}_{\boldsymbol{s l t a s k}\left(\mathbf{1}, l_{\boldsymbol{x}}\right)}$. And we have $\tau>L_{s_{x}-1}$ by the following.

- If $x$ is in the first row in $M_{s}$, then $\tau>L_{s_{x}-1}$ because $x \notin \operatorname{sltask}\left(1, s_{x}-1\right)$ and the task in the first row uses a new time unit after time $L_{s_{x}-1}$.

- If $x$ is in row $r$ such that $r \geq 2$, i.e., $x=M_{s}[r, \tau]$, then $M_{s}[1, \tau] \notin \operatorname{sltask}\left(1, s_{x}-\right.$ 1) because $M_{s}[1, \tau]$ and $x$ overlap by Fact 1 and thus $\tau>L_{s_{x}-1}$.

Hence $L_{s_{x}-1}+1 \leq \tau \leq L_{l_{x}}$. Since $x$ is adjacent to all $t$ such that $m L_{s_{x}-1}+1 \leq$ $t \leq m L_{l_{x}}$ in $E_{b}$, every edge $(x, j)$ in $E^{\prime}$ is also in $E_{b}$. Since $j$ 's are distinct, $E^{\prime}$ is a maximum matching of size $n$ in $G_{b}$.

Lemma 5. The $m \times L_{k}$ matrix $M_{b}$ is an optimal schedule for $\boldsymbol{G}_{\boldsymbol{I}}$.

Proof. Consider tasks $x$ and $y$ of $\boldsymbol{G}_{\boldsymbol{I}}$ such that $y$ is a successor of $x$. Let $\tau$ and $\tau^{\prime}$ be the columns of $M_{b}$ at which $x$ and $y$ are, respectively. Note that $M_{b}$ has $L_{k}$ columns, which is opt $(I)$, and all tasks are in $M_{b}$ by Lemma 4. Since all the tasks in the same column of $M_{b}$ are independent of each other in $\boldsymbol{G}_{\boldsymbol{I}}$ by Lemma 3, we can prove that $M_{b}$ is an optimal schedule for $\boldsymbol{G}_{\boldsymbol{I}}$ by showing that $\tau^{\prime}>\tau$.

Let $t$ and $t^{\prime}$ be integers matched with $x$ and $y$, respectively, in the maximum matching of $G_{b}$. Then $t \leq m L_{l_{x}}$ and $m L_{s_{y}-1}+1 \leq t^{\prime}$ by definition of $G_{b}$. Since $y$ is a successor of $x$, we have $l_{x}<s_{y}$ by Lemma 2, which implies that $t^{\prime}$ is greater than $t$. Since $y$ must be in a different column of $M_{b}$ with that of $x$ by Lemma 3 , we have $\tau^{\prime}>\tau$.

Theorem 2. An optimal schedule for $\boldsymbol{G}_{\boldsymbol{I}}$ on $m$ processors can be solved in $O\left(\log ^{2} v+(n \log n) / v\right)$ time with $O\left(n v^{2}+n^{2}\right)$ operations on the CREW PRAM, where $v \leq n$ is a parameter. 
Proof. The correctness of algorithm m-schedule follows from Lemma 5 We will show that m-schedule takes $O\left(\log ^{2} v+(n \log n) / v\right)$ time and $O\left(n v^{2}+n^{2}\right)$ operations on the CREW PRAM. Step 1 takes $O(\log n)$ time using $O(n \log n)$ operations as follows. In a sorted endpoints sequence, put 1 at the right endpoints of Lemma 1 and 0 in other endpoints and compute $s_{x}$ and $l_{x}$ using a prefix sum, i.e., the prefix sum at $x . l$ is $s_{x}-1$ and the prefix sum at $x . r$ is $l_{x}$. Since we can compute all $L_{j}(=\operatorname{len}(1, j))$ for $1 \leq j \leq k$ by running algorithm m-length in Section 4 only once, Step 2 takes $O\left(\log ^{2} v+(n \log n) / v\right)$ time with $O\left(n v^{2}+n^{2}\right)$ operations. Step 3 takes constant time using $O(n)$ operations. Step 4 takes $O\left(\log ^{2} n\right)$ time with $O\left(n \log ^{2} n\right)$ operations using Dekel and Sahni's algorithm [5.

\section{Computing the Length of an Optimal Schedule}

We now describe our $m$-LOS algorithm. We obtain our $m$-LOS algorithm in Figure 3 by generalizing the 2-LOS algorithm in 2 .

Algorithm m-length

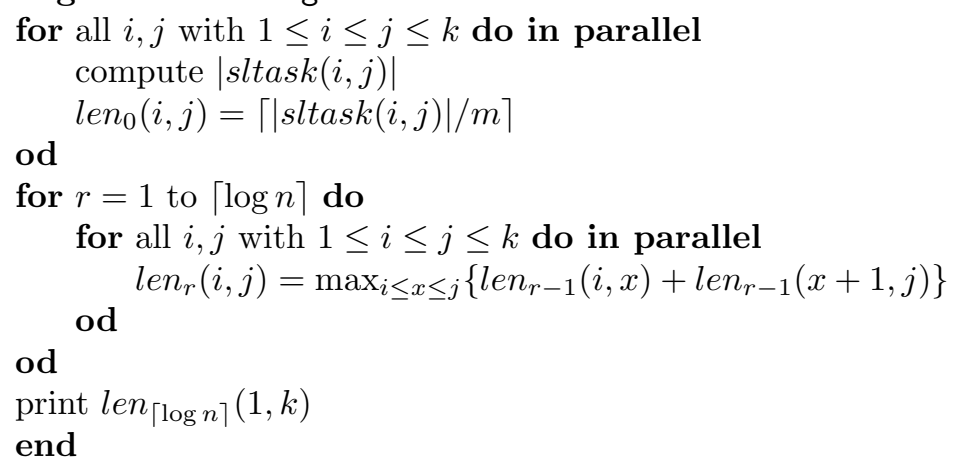

Fig. 3. An efficient $m$-LOS algorithm

We now prove the correctness of algorithm m-length. We first define sets $\chi_{1}, \ldots, \chi_{z}$ of tasks for an interval order such that:

- all tasks in any $\chi_{i+1}$ are predecessors of all tasks in $\chi_{i}$ and

- the length of an optimal schedule equals $\sum_{i}\left\lceil\left|\chi_{i}\right| / m\right\rceil$.

Our sets $\chi_{i}$ 's for $m$-processor scheduling are the generalization of those for two-processor scheduling [3] tailored to the special case of interval orders. We do not explicitly compute these sets in algorithm m-length in Figure 3; we only make use of them for the proof of its correctness.

We define the sets $\chi_{1}, \ldots, \chi_{z}$ of tasks from the schedule $M_{s}$ computed by algorithm m-seq in Figure 1 as follows. We recursively define tasks $v_{i}$ and $w_{i}$ for $i \geq 1$. Let $v_{1}$ be the last task executed by processor $P_{1}$ (i.e., $v_{1}$ is $M_{s}[1$, opt $(I)]$ ) and $w_{1}$ is (a possibly empty task) $M_{s}[m$,opt $(I)]$. Given $v_{i}$, we define $w_{i+1}$ and $v_{i+1}$ as follows. Suppose that $v_{i}$ is $M_{s}[1, \tau]$. Let $\tau^{\prime}$ be the largest column number 
less than $\tau$ in $M_{s}$ such that $M_{s}\left[m, \tau^{\prime}\right] . r>v_{i} . r$ or $M_{s}\left[m, \tau^{\prime}\right]$ is an empty task. Then $w_{i+1}$ is $M_{s}\left[m, \tau^{\prime}\right]$ and $v_{i+1}$ is $M_{s}\left[1, \tau^{\prime}\right]$. In Figure 2$] v_{1}=o$, and thus $w_{2}$ is an empty task and $v_{2}=i$. Also $w_{3}=j$ and $v_{3}=c$. Note that each column $\tau^{\prime \prime}$ such that $\tau^{\prime}<\tau^{\prime \prime}<\tau$ is full. Let $z$ be the largest index for which $w_{z}$ and $v_{z}$ are defined. We assume that $v_{z+1}$ is a special interval $\beta$ whose right endpoint is smaller than all endpoints in $I$ and $l_{v_{z+1}}=0$. Let $\tau_{i}, 1 \leq i \leq z$, denote the timestep at which $v_{i}$ is executed. Define $\chi_{i}$ to be $\left\{x \mid x\right.$ is in column $\tau^{\prime \prime}$ such that $\left.\tau_{i+1}<\tau^{\prime \prime}<\tau_{i}\right\} \cup\left\{v_{i}\right\}$. In Figure 2, sets $\chi_{i}$ 's for $\boldsymbol{G}_{\boldsymbol{I}}$ are marked by thick lines in the schedule. The characteristics of $\chi_{i}$ 's are as follows.

Lemma 6. In $\boldsymbol{G}_{\boldsymbol{I}}$, every task $x \in \chi_{i}$ satisfies $x . r \leq v_{i} . r$.

Proof. Since $\tau_{i+1}$ is the largest column number less than $\tau_{i}$ such that $M_{s}\left[m, \tau_{i+1}\right.$ ]. $r>v_{i} . r$, we have $M_{s}\left[m, \tau^{\prime \prime}\right] . r<v_{i} . r$ for $\tau_{i+1}<\tau^{\prime \prime}<\tau_{i}$. Note that we assume that an empty task has the largest right endpoint in $I$. Since the task in the last row in each column has the largest right endpoint in the column by Fact 2 , every task $x$ in column $\tau^{\prime \prime}$ such that $\tau_{i+1}<\tau^{\prime \prime}<\tau_{i}$ satisfies $x . r<v_{i} . r$. Therefore, every $x \in \chi_{i}$ satisfies $x . r \leq v_{i} . r$.

Lemma 7. In $\boldsymbol{G}_{\boldsymbol{I}}$, all tasks in $\chi_{i+1}$ are predecessors of all tasks in $\chi_{i}$.

Proof. Let $y$ be a task in $\chi_{i}$. Since every $x \in \chi_{i+1}$ satisfies $x . r \leq v_{i+1} . r$ by Lemma 6] we can prove the lemma by showing that $v_{i+1} . r<y$.l. Since $y . r \leq v_{i} . r$ and $v_{i} . r<w_{i+1} . r=M_{s}\left[m, \tau_{i+1}\right] . r$, we have $y . r<M_{s}\left[m, \tau_{i+1}\right] . r$. Since $y$ is at one of columns $\tau_{i+1}+1, \ldots, \tau_{i}$, we have $M_{s}\left[1, \tau_{i+1}\right] . r<y . r$ by Facts 2 and 3 . Hence $M_{s}\left[1, \tau_{i+1}\right] . r<y . r<M_{s}\left[m, \tau_{i+1}\right] . r$. If $y$ overlaps $M_{s}\left[1, \tau_{i+1}\right]=v_{i+1}$, then $y$ should be assigned to column $\tau_{i+1}$ in $\mathrm{m}$-seq in Figure 1 which is a contradiction. Therefore, $v_{i+1} . r<y . l$.

Theorem 3. The length of an optimal schedule for $\boldsymbol{G}_{\boldsymbol{I}}$ is $\sum_{1 \leq i \leq z}\left\lceil\left|\chi_{i}\right| / m\right\rceil$.

Proof. Since each column $\tau^{\prime \prime}$ such that $\tau_{i+1}<\tau^{\prime \prime}<\tau_{i}$ is full and $v_{i}=M_{s}\left[1, \tau_{i}\right]$ is in $\chi_{i}$, we get $\left\lceil\left|\chi_{i}\right| / m\right\rceil=\tau-\tau^{\prime}$. Therefore, $\sum_{1 \leq i \leq z}\left\lceil\left|\chi_{i}\right| / m\right\rceil$ is the number of columns in $M_{s}$, which is $\operatorname{opt}(I)$.

When $m=2$, Chung et al. [2] showed that $\chi_{i}$ equals $\operatorname{sltask}\left(l_{v_{i+1}}+1, l_{v_{i}}\right)$ for $1 \leq i \leq z$ and that $\operatorname{len} n_{\lceil\log n\rceil}(i, j)$ equals $\operatorname{len}(i, j)$ for $1 \leq i \leq j \leq k$. Similarly, we can prove the correctness of algorithm m-length as follows.

Lemma 8. In $\boldsymbol{G}_{\boldsymbol{I}}, \chi_{i} \subseteq \operatorname{sltask}\left(l_{v_{i+1}}+1, l_{v_{i}}\right)$ for $1 \leq i \leq z$.

Proof. Let $x$ be a task in $\chi_{i}$. Since $v_{i+1} \in \chi_{i+1}$ is a predecessor of $x$ by Lemma[7] we have $v_{i+1} . r<x . l$, which implies $l_{v_{i+1}}<s_{x}$ by Lemma 2 , Since $x . r \leq v_{i} . r$ by Lemma 6, we have $l_{x} \leq l_{v_{i}}$. Therefore, $x$ is in $\operatorname{sltask}\left(l_{v_{i+1}}+1, l_{v_{i}}\right)$.

Corollary 1. In $\boldsymbol{G}_{\boldsymbol{I}}, \bigcup_{i \leq t \leq j} \chi_{t} \subseteq \operatorname{sltask}\left(l_{v_{j+1}}+1, l_{v_{i}}\right)$ for $1 \leq i \leq j \leq z$. 
Corollary 2. In $\boldsymbol{G}_{\boldsymbol{I}}$, all tasks in $\operatorname{stask}\left(l_{v_{j+1}}+1, l_{v_{i}}\right), i \leq j$, are successors of all tasks in $\bigcup_{j+1 \leq t \leq z} \chi_{t}$ and predecessors of all tasks in $\bigcup_{1 \leq t \leq i-1} \chi_{t}$.

Lemma 9. Every task in sltask $\left(l_{v_{i+1}}+1, l_{v_{i}}\right)$ is in one of columns $\tau_{i+1}+$ $1, \ldots, \tau_{i}$.

Proof. Let $y$ be a task in $\operatorname{sltask}\left(l_{v_{i+1}}+1, l_{v_{i}}\right)$. Note that $y$ satisfies $M_{s}\left[1, \tau_{i+1}\right] . r<$ $y . l$ by Lemma 2 and $y . r<M_{s}\left[1, \tau_{i}+1\right] . l$ by Lemma 7 . Therefore, $y$ must be in one of columns $\tau_{i+1}+1, \ldots, \tau_{i}$ by the way algorithm m-seq in Figure 1 works.

Lemma 10. In $\boldsymbol{G}_{\boldsymbol{I}}, \sum_{i \leq t \leq j}\left\lceil\left|\chi_{t}\right| / m\right\rceil=\operatorname{len}\left(l_{v_{j+1}}+1, l_{v_{i}}\right)$ for $1 \leq i \leq j \leq z$.

Proof. The proof for the case $m=2$ is in Lemma 8 in [2] and the proof of the lemma is similar.

Lemma 11. In algorithm m-length, $\operatorname{len}_{r}(i, j) \leq \operatorname{len}(i, j)$ for $0 \leq r \leq\lceil\log n\rceil$.

Proof. It is similar to the proof of Lemma 9 in [2].

Lemma 12. In algorithm $\mathrm{m}$-length, $\operatorname{len} n_{\lceil\log n\rceil}(i, j) \geq \operatorname{len}(i, j)$ for $1 \leq i \leq j \leq k$.

Proof. We show that $l e n_{\lceil\log n\rceil}(1, k) \geq \operatorname{len}(1, k)$. We prove by induction on $r$ that for $i \leq 2^{r}$,

$$
\operatorname{len}_{r}\left(l_{v_{x+i}}+1, l_{v_{x}}\right) \geq \sum_{x \leq t<x+i}\left\lceil\left|\chi_{t}\right| / m\right\rceil
$$

When $r=0$, (1) holds as follows. Since each column $\tau^{\prime \prime}$ such that $\tau_{i+1}<\tau^{\prime \prime}<\tau_{i}$ is full, $\left\lceil\left|\operatorname{sltask}\left(l_{v_{x+1}}+1, l_{v_{x}}\right)\right| / m\right\rceil \geq\left\lceil\left|\chi_{x}\right| / m\right\rceil \geq \tau_{i}-\tau_{i+1}$ by Lemma 8. Since $\left\lceil\left|\operatorname{sltask}\left(l_{v_{x+1}}+1, l_{v_{x}}\right)\right| / m\right\rceil \leq \tau_{i}-\tau_{i+1}$ by Lemma 9, we have $\left\lceil\mid \operatorname{sltask}\left(l_{v_{x+1}}+\right.\right.$ $\left.\left.1, l_{v_{x}}\right) \mid / m\right\rceil=\tau_{i}-\tau_{i+1}$. Therefore, $\operatorname{len}_{0}\left(l_{v_{x+1}}+1, l_{v_{x}}\right)=\left\lceil\left|\operatorname{sltask}\left(l_{v_{x+1}}+1, l_{v_{x}}\right)\right| / m\right\rceil$ $=\left\lceil\left|\chi_{x}\right| / m\right\rceil$. Assume that (10) holds after $r$ iterations of the main loop. In the $(r+1)$ st iteration for $2^{r}<i \leq 2^{r+1}$,

$$
\begin{aligned}
\operatorname{len}_{r+1}\left(l_{v_{x+i}}+1, l_{v_{x}}\right) & \geq \operatorname{len}_{r}\left(l_{v_{x+i}}+1, l_{v_{x+2^{r}}}\right)+\operatorname{len}_{r}\left(l_{v_{x+2^{r}}}+1, l_{v_{x}}\right) \\
& \geq \sum_{x+2^{r} \leq t<x+i}\left\lceil\left|\chi_{t}\right| / m\right\rceil+\sum_{x \leq t<x+2^{r}}\left\lceil\left|\chi_{t}\right| / m\right\rceil \\
& \geq \sum_{x \leq t<x+i}\left\lceil\left|\chi_{t}\right| / m\right\rceil
\end{aligned}
$$

Since each $\chi_{i}$ contains at least one task, there are at most $n \chi_{i}$ 's. Thus,

$$
\begin{aligned}
\operatorname{len}_{\lceil\log n\rceil}\left(l_{v_{z+1}}+1, l_{v_{1}}\right) & \geq \sum_{1 \leq t \leq z}\left\lceil\left|\chi_{t}\right| / m\right\rceil \\
& \geq \operatorname{len}\left(l_{v_{z+1}}+1, l_{v_{1}}\right) \quad \text { by Lemma 10. }
\end{aligned}
$$

Since $l_{v_{z+1}}+1=1$ and $l_{v_{1}}=k$, we get $\operatorname{len} n_{\lceil\log n\rceil}(1, k) \geq \operatorname{len}(1, k)$. Similarly, we can prove that $\operatorname{len} n_{\lceil\log n\rceil}(i, j) \geq \operatorname{len}(i, j)$ for $1 \leq i \leq j \leq k$ by using sets of $\chi_{i}$ 's for $\boldsymbol{G}_{\boldsymbol{I}^{\prime}}$, where $I^{\prime}$ is $\operatorname{sltask}(i, j)$ in $I$. 
Theorem 4. There is an $m$-LOS algorithm that requires $O\left(\log ^{2} v+(n \log n) / v\right)$ time and $O\left(n v^{2}+n^{2}\right)$ operations on the CREW PRAM, where $v$ is a parameter such that $v \leq n$. Furthermore, it also computes the length of an optimal schedule for $\boldsymbol{G}_{\text {sltask }(1, j)}, 1 \leq j \leq k$.

Proof. The correctness of algorithm m-length follows from Lemmas 11 and 12 Algorithm m-length has a straightforward implementation using $O\left(\log ^{2} n\right)$ time and $O\left(n^{3}\right)$ processors on the CREW PRAM. It can be improved to $O\left(\log ^{2} v+\right.$ $(n \log n) / v)$ time and $O\left(n v^{2}+n^{2}\right)$ operations using Galil and Park's reduction technique [8], which is similar to the proof of Theorem 3 in [2].

\section{References}

1. M. Bartusch, R. H. Mohring, and F. J. Radermacher, "M-machine unit time scheduling: A report of ongoing research," Lecture Notes in Economics and Mathematical Systems 304, Springer-Verlag (1988) 165-212.

2. Y. Chung, K. Park, and Y. Cho, "Parallel maximum matching algorithms in interval graphs," Int'l. J. Foundations of Comput. Science 10, 1 (1999) 47-60.

3. E. Coffman and R. Graham, "Optimal scheduling for two processor systems," Acta Informatica 1 (1972) 200-213.

4. R. Cole, "Parallel merge sort," SIAM J. Comput. 17, 4 (1988) 770-785.

5. E. Dekel and S. Sahni, "A parallel matching algorithm for convex bipartite graphs and applications to scheduling," J. Parallel Distrib. Comput. 1 (1984) 185-205.

6. M. Fujii, T.Kasami, and K. Ninomiya, "Optimal sequencing of two equivalent processors," SIAM J. Appl. Math. 17 (1969) 784-789.

7. H.N. Gabow, "An almost-linear algorithm for two-processor scheduling," J. ACM 29, 3 (1982) 766-780.

8. Z. Galil and K. Park, "Parallel algorithms for dynamic programming recurrences with more than $O(1)$ dependency," J. Parallel Distrib. Comput. 21, (1994) 213222 .

9. M. R. Garey and D. S. Johnson, "Scheduling tasks with nonuniform deadlines on two processors," J. ACM 23 (1976) 461-467.

10. M.C. Golumbic, Graph theory and perfect graphs, Academic Press, New York, 1980.

11. D. Helmbold and E. Mayr, "Two processor scheduling is in NC," SIAM J. Comput. 16, 4 (1987) 747-759.

12. T. C. Hu, "Parallel sequencing and assembly line problems," Oper. Res. 9 (1961) 841-848.

13. E. L. Lawler, J. K. Lenstra, A. H. G. R. Kan, and D. B. Shmoys, "Sequencing and scheduling: Algorithms and complexity," Technical report, Centrum voor Wiskunde en Informatica, 1989.

14. E. Mayr, "Scheduling interval orders in parallel," Parallel Algorithms and Applications 8 (1996) 21-34.

15. C. H. Papadimitriou and M Yannakakis, "Scheduling interval-ordered tasks," SIAM J. Comput. 8 (1979), pp. 405-409.

16. H. Jung, M. Serna and P. Spirakis, "A parallel algorithm for two processors precedence constraint scheduling," Proc. Int'l Colloquium on Automata, Languages and Programming (1991) pp. 417-428.

17. D. Kozen, U.V. Vazirani and V.V. Vazirani, " $N C$ algorithms for cocomparability graphs, interval graphs, and unique perfect matchings," Proc. Foundations of Software Technology and Theoretical Computer Science (1985) pp. 496-503. 
18. A. Moitra and R. Johnson, "A parallel algorithm for maximum matching on interval graphs," Proc. Int'l Conference on Parallel Processing 3 (1989) pp. 114-120.

19. S. Sunder and X.He, "Scheduling interval ordered tasks in parallel," J. Algorithm 26 (1998), pp.34-47.

20. J. D. Ulman, Complexity of sequencing problems, in "Computer and job scheduling theory" (E. G. Coffman, Ed.), Wiley, 1976.

21. U.V. Vazirani and V.V. Vazirani, "The two processor scheduling is in random $N C$," SIAM J. Comput. (1989) pp. 1140-1148. 